\title{
Orientamento imprenditoriale e performance: un'indagine tra le università italiane
}

\author{
Angelo RivieZzo ${ }^{*}$ Maria Rosaria Napolitano **
}

\begin{abstract}
Obiettivo del paper: Il lavoro si propone di misurare empiricamente l'orientamento imprenditoriale dei dipartimenti delle università italiane e verificare l'impatto che esso ha sulla capacità di generare brevetti e imprese spin-off della ricerca.

Metodologia: È stato inviato un questionario web-based a 238 direttori di dipartimento, ottenendo 103 risposte utili. Un'analisi di regressione multipla ha poi consentito di indagare il legame tra l'orientamento imprenditoriale e le performance dei dipartimenti.

Risultati: L'orientamento imprenditoriale impatta significativamente sulla capacità dei dipartimenti universitari di generare brevetti e imprese spin-off della ricerca. Tuttavia non tutte le variabili utilizzate per misurare il costrutto teorico, derivate da un precedente studio, rivestono lo stesso ruolo.

Limiti della ricerca: Un limite dello studio è rappresentato dalla numerosità del campione ottenuto e dal fatto che l'indagine è concentrata solo in Italia. Inoltre, per l'analisi statistica dei risultati si sarebbero potuti impiegare approcci più evoluti.

Implicazioni pratiche: Il lavoro integra il tradizionale approccio alla valutazione della capacità imprenditoriale delle università che si limita a considerare i risultati, ma spesso non ne indaga le determinanti strategiche. Inoltre individua i fattori sui quali occorre intervenire, a livello di singoli dipartimenti o di ateneo, per sostenere l'imprenditorialità accademica.

Originalità del paper: La letteratura esistente sul tema dell'università imprenditoriale ha tradizionalmente approfondito le implicazioni organizzative del nuovo modello rispetto a quelle strategiche. Il lavoro considera l'orientamento strategico come un fattore decisivo per l'imprenditorialità accademica, misurandone empiricamente l'impatto.
\end{abstract}

Parole chiave: orientamento imprenditoriale; imprenditorialità accademica; terza missione

Purpose of the paper: The paper aims at empirically measuring the entrepreneurial orientation of the Italian universities' departments and the impact it has on their ability to generate patents and spin-offs from research.

Methodology: We sent a web-based questionnaire to 238 Heads of department of Italian universities, getting 103 useful responses. Through a multiple regression analysis we investigated the link between departments' entrepreneurial orientation and performance.

\footnotetext{
Ricercatore di Economia e Gestione delle Imprese - Università degli Studi del Sannio e-mail: angelo.riviezzo@unisannio.it

** Ordinario di Economia e Gestione delle Imprese - Università degli Studi del Sannio e-mail: napolitano@unisannio.it
} 
Findings: The entrepreneurial orientation significantly affects the ability of university departments to generate patents and spin-offs. However, not all the dimensions we used to operationalize the theoretical construct that we derived from a previous study play the same role.

Research limits: A limitation of the study is the sample size obtained and the fact that the survey was administered just in Italy. Furthermore, for the statistical analysis of results we could have used more advanced approaches.

Practical implications: The paper integrates the traditional approach in assessing the entrepreneurial ability of universities, which is limited to the focus on the results, without investigating their strategic determinants. Furthermore, it identifies the factors on which is possible to act, at the level of single departments or the whole university, in order to foster academic entrepreneurship.

Originality of the paper: The literature on entrepreneurial university has traditionally studied the organizational implications of the new model rather than the strategic implications. This paper considers the strategic orientation as a key factor to academic entrepreneurship, empirically measuring its impact.

Key words: entrepreneurial orientation; academic entrepreneurship; third mission

\section{Introduzione}

L'orientamento strategico ispira la strategia di un'organizzazione e ne definisce l'identità in termini d'idee, convinzioni, atteggiamenti, valori, rapporti con gli stakeholder esterni e obiettivi da perseguire (Coda, 1988; Venkatraman, 1989). In linea con precedenti contributi (Rebora, 2008; Todorovic et al., 2011), il presente lavoro mira ad adottare siffatta prospettiva nel contesto specifico delle università con l'obiettivo di analizzare le determinanti dell'imprenditorialità a livello accademico. In particolare, mutuando un approccio tipicamente utilizzato negli studi sulle imprese, l'attenzione è focalizzata sull'orientamento imprenditoriale (Miller, 1983; Covin e Slevin, 1986; Lumpkin e Dess, 1996) e sull'impatto che esso ha sulla capacità delle università di valorizzare a fini commerciali i risultati della propria attività di ricerca.

Come noto, nel corso degli anni l'università è stata chiamata a promuovere una trasformazione del proprio ruolo da organizzazione votata alla produzione di conoscenza a organizzazione attivamente coinvolta anche nella diffusione e valorizzazione della stessa conoscenza (Etzkowitz, 1983, 2004; Clark, 1998; Gibb, 2005; Piccaluga, 2001; Compagno e Pittino, 2006). In altri termini, si è affermato un nuovo modello di "università imprenditoriale" (Etzkowitz, 1983), caratterizzato dall'essere non più "an isolated island of knowledge" (Klofsten e Jones-Evans, 2000), bensì "an economic actor on its own right" (Etzkowitz, 2004) con un impegno diretto per il progresso economico e sociale. Come evidenziato dalla copiosa letteratura sul tema, tale "rivoluzione accademica" è in corso in tutto il mondo, ancorché a un difforme stadio di evoluzione.

Nel nostro Paese, ad esempio, le evidenze empiriche sembrano mostrare una generalizzata accettazione e una più positiva propensione verso l'imprenditorialità 
accademica con risultati che cominciano a essere significativi, seppur ancora lontani da quelli delle università nordeuropee o nordamericane (Cesaroni et al., 2005; Piccaluga e Balderi, 2006; Napolitano e Riviezzo, 2008; Baglieri, 2008; Riviezzo e Napolitano, 2010; CNVSU, 2011; Netval, 2011, 2012, 2013; Balderi et al., 2012). Gli atenei italiani hanno incrementato la propria capacità di attrarre finanziamenti esterni (CNVSU, 2011); buona parte di essi ha introdotto nella propria struttura organizzativa delle unità dedicate al trasferimento tecnologico (Riviezzo e Napolitano, 2010; Balderi et al., 2012); il numero d'imprese spin-off create, di brevetti detenuti e di licenze concesse è costantemente e significativamente cresciuto negli anni (Netval, 2011, 2012, 2013).

Il modello di università imprenditoriale richiede numerosi cambiamenti, in termini strategici, organizzativi e culturali. Molta attenzione da parte degli studiosi ha calamitato la prospettiva organizzativa, con un'approfondita analisi delle innovazioni introdotte, dei fattori che hanno causato e accelerato la trasformazione e delle variabili interne ed esterne in grado di condizionarne gli esiti. Una certa enfasi è stata conferita pure alla prospettiva culturale, valutando le possibili difficoltà di dialogo dell'accademia con il mondo produttivo e le implicazioni che ne derivano per l'efficacia del trasferimento tecnologico. Ad oggi, sembra invece aver suscitato minor interesse la prospettiva strategica, privilegiata nel presente lavoro, che si propone di misurare empiricamente l'orientamento imprenditoriale dei dipartimenti delle università italiane e verificare l'impatto che esso ha sulla capacità di generare brevetti e imprese spin-off della ricerca.

A tal fine, si è partiti da un'innovativa declinazione del costrutto teorico di orientamento imprenditoriale, recentemente sviluppata da alcuni studiosi canadesi (Todorovic et al., 2011) per tener conto delle specificità delle università rispetto alle imprese profit-oriented. Adottando il medesimo approccio metodologico nella misurazione dell'imprenditorialità e delle performance dei dipartimenti, il presente lavoro mira a comparare i risultati ottenuti in Italia con quelli dello studio condotto in Canada, configurandosi pertanto come un replication study. L'indagine è stata sviluppata attraverso l'invio di un questionario web-based a 238 direttori di dipartimento di oltre 30 università italiane, e ha ottenuto 103 risposte utili. Il legame tra l'orientamento e le performance imprenditoriali dei dipartimenti universitari italiani è stato indagato attraverso un'analisi di regressione multipla.

Nei paragrafi seguenti si propone una sintetica review della letteratura sul modello di università imprenditoriale e sul concetto di orientamento imprenditoriale. In seguito s'illustrano la metodologia utilizzata e i risultati ottenuti. Infine, sono discusse le implicazioni e le limitazioni dello studio.

\section{Analisi della letteratura}

\subsection{II concetto di università imprenditoriale}

Particolarmente ricca è la letteratura sul modello di università imprenditoriale, sebbene non sia tuttora possibile identificare delle precise connotazioni che lo 
contraddistinguano in maniera univoca. È stato notato che le università diventano imprenditoriali quando (Clark, 1998) "they are unafraid to maximise the potential for commercialisation of their ideas and create value in society and do not see this as a significant threat to academic values". In tal senso, aprendosi all'esterno e instaurando relazioni con gli stakeholder rilevanti in modo sia formale che informale, cercano di divenire delle 'stand-up' universities (Clark, 1998) e di assumere "a pro-active stance in putting knowledge to use" (Etzkowitz, 1983).

Numerosi sono i contributi incentrati sull'analisi delle motivazioni che hanno condotto a un'intensificazione dei rapporti tra l'accademia e il mondo produttivo. Tra i fattori maggiormente discussi è possibile, ad esempio, annoverare: lo sviluppo tecnologico e il contenuto sempre più tecnico di tutti i prodotti; la necessità delle università di diversificare le proprie entrate per far fronte ad una progressiva diminuzione dei finanziamenti pubblici; la crescente autonomia e complessità delle istituzioni di ricerca; la maggior pressione politica per avere ritorni economici dalla ricerca; l'emergere di nuove discipline, frutto della combinazione o fusione di discipline pre-esistenti (biotecnologie, bioinformatica etc.) (Geuna, 1998; Piccaluga, 2001; Bencardino e Napolitano, 2011).

Molta attenzione è stata conferita allo studio dell'evoluzione organizzativa delle università impegnate nell'implementazione della "terza missione" dello sviluppo economico e sociale. È stata in tal senso evidenziata l'eterogeneità delle soluzioni sperimentate (Hussler et al., 2010). Etzkowitz (2004) descrive il percorso evolutivo che accomuna molti dei casi direttamente osservati in vari paesi del mondo: si parte con l'istituzione di un Industrial Liaison Office (ILO), responsabile della promozione di occasioni di interazione tra i ricercatori e le imprese; si continua con la creazione di un Technology Transfer Office (TTO), incaricato di identificare, brevettare e commercializzare le invenzioni di maggior interesse; ed infine si giunge alla realizzazione di un Incubatore, che facilita la possibilità di trasferire la conoscenza verso l'esterno nella forma di nuove imprese create dagli inventori. Non necessariamente, tuttavia, esiste una così netta distinzione nella natura e nella funzione delle unità organizzative. Nel caso specifico dell'Italia, ad esempio, recenti studi (Riviezzo e Napolitano, 2010; Netval, 2011, 2012; Balderi et al., 2012) mostrano come gli uffici predisposti negli ultimi anni per implementare la terza missione siano mediamente molto piccoli e assolvano nella maggior parte dei casi sia alle funzioni tipiche degli ILO che dei TTO. Ancora piuttosto scarsa è inoltre la diffusione presso le università del nostro Paese degli Incubatori (Schillaci et al., 2008; Riviezzo e Napolitano, 2010). In un'interessante applicazione della catena del valore di Porter al processo di valorizzazione della ricerca scientifica, Compagno e Pittino (2006) riconducono tali entità organizzative alle attività di supporto a sostegno delle attività primarie che sono: il riconoscimento delle opportunità, soltanto implicite nei risultati della ricerca, e la loro traduzione in una forma suscettibile di essere commercializzata; la formalizzazione di un piano per realizzare il valore dell'idea innovativa; l'ottenimento di un brevetto che, spesso, può costituire le fondamenta su cui creare uno spin-off. 
Parallelamente all'evoluzione organizzativa, molti autori evidenziano la possibile progressione nel corso del tempo nella natura delle relazioni tra università e imprese, da singole e occasionali transazioni a rapporti di lungo termine (Bercovitz e Feldman, 2006). Questa evoluzione è tuttavia influenzata dal contesto in cui si sviluppano le relazioni: sia le condizioni esterne all'università che quelle interne influiscono infatti sull'efficacia e sui risultati del trasferimento tecnologico.

Cambiamenti significativi nel quadro normativo, economico o politico possono accelerare la trasformazione della ricerca scientifica in prodotti commerciali. Un esempio ben noto e frequentemente citato in letteratura è il Bayh-Dole Act americano del 1980 (Powers e McDougall, 2005; Lerner, 2005). Allo stesso modo, le caratteristiche delle imprese locali assumono un ruolo rilevante nel determinare la relazione con l'università come partner strategico (Cesaroni et al., 2005; Bercovitz e Feldman, 2006). Ancora, il livello d'investimenti pubblici e privati in ricerca e la presenza di investitori formali e informali - merchant bank, venture capital, business angels, etc. - può influire sull'attività imprenditoriale sviluppata all'interno delle università (Roberts e Malone, 1996).

Non tutte le università che sviluppano attività di ricerca sono state abili a generare effetti economici sul territorio (Feller, 1990). Ci sono, infatti, anche fattori interni che caratterizzano l'ambiente universitario e che determinano il livello di trasferimento tecnologico realizzato. A tal proposito, Gras et al. (2008) individuano cinque categorie di risorse e competenze interne che risultano determinanti: la presenza di politiche e strategie per il trasferimento tecnologico; il capitale umano; lo stock di tecnologie detenuto; le risorse e competenze degli uffici per il trasferimento tecnologico; le misure e le politiche di supporto.

Un'altra prospettiva d'analisi molto utilizzata è quella che si focalizza sugli inventori accademici, indagandone le intenzioni (Prodan e Drnovsek, 2010), la propensione a dedicarsi ad attività commerciali (Hoye e Pries, 2009) o le difficoltà di accesso al capitale per finanziare lo start-up (Macho-Stadler et al., 2008; Gurdon e Samsom, 2010). Altri studiosi si soffermano sulle differenze culturali tra l'università e il mondo delle imprese, indagandone gli effetti sul processo di trasferimento tecnologico (Liyanage e Mitchell, 1994; van Geenhuizen e Soetanto, 2009).

Un'ulteriore peculiarità del modello di università imprenditoriale è rappresentata dalla creazione e promozione della cultura imprenditoriale. In tal senso, tale modello si caratterizza anche per la diffusione d'insegnamenti dedicati all'imprenditorialità in tutte le aree disciplinari (Clark, 1998; Van Burg et al., 2008). Recenti indagini nel nostro Paese (Riviezzo e Napolitano, 2010) testimoniano come, anche da questo punto di vista, la situazione sia in netto miglioramento, ma ancora ben distante da quella di paesi con maggior tradizione.

Occorre infine evidenziare come parte della letteratura assuma una posizione critica rispetto all'inclusione della terza missione tra quelle di cui l'università è portatrice (ad esempio, Slaughter e Leslie, 1997; Hayes e Wynyard, 2002; Barnett, 2003; Bok, 2003). Le opposizioni alla "McUniversity" (Hayes e Wynyard, 2002) e al "capitalismo accademico" (Slaughter e Leslie, 1997) si basano sull'assunto che 
esista un conflitto per il ricercatore tra la missione della ricerca e l'impegno per lo sfruttamento a fini commerciali dei risultati scientifici. Come notato da Etzkowitz (1998), tuttavia, ci si può attendere che - così come la ricerca è stata integrata tra le funzioni universitarie per effetto della "prima rivoluzione accademica" alla fine del diciannovesimo secolo - allo stesso modo la "seconda rivoluzione accademica" porterà la terza missione ad essere integrata rispetto alla didattica e alla ricerca, "with incubators adjoining classrooms and laboratory facilities".

\subsection{Il concetto di orientamento imprenditoriale e la sua applicazione alle università}

Sebbene nel linguaggio comune il fenomeno imprenditoriale sia generalmente ricondotto alla creazione e all'avvio di nuove imprese, la ricca letteratura esistente sul tema della corporate entrepreneurship evidenzia l'importanza dell'imprenditorialità nell'ambito di organizzazioni già esistenti. L'orientamento imprenditoriale rappresenta il modo in cui è possibile rilevare l'imprenditorialità nella sua essenza, in quanto identifica i processi, i metodi e gli stili decisionali delle imprese che agiscono "imprenditorialmente".

Si tratta di un costrutto multidimensionale che la letteratura tradizionalmente riconduce a tre elementi: innovatività, propensione al rischio e proattività (Miller, 1983; Covin e Slevin, 1986). Una più ricca declinazione prevede due ulteriori dimensioni: aggressività competitiva e autonomia (Lumpkin e Dess, 1996). L'innovatività attiene non solo ai prodotti o servizi e ai mercati, ma anche alle tecnologie, ai sistemi amministrativi e alle strategie. La propensione al rischio trova espressione nella volontà di allocare risorse finanziarie a progetti dall'esito incerto, circostanza inscindibilmente legata al continuo perseguimento di nuove opportunità. La proattività esprime la necessità di anticipare i bisogni dei clienti per conseguire un vantaggio competitivo nei confronti di una concorrenza sempre più agguerrita. L'aggressività competitiva misura la propensione dell'impresa ad affrontare i propri concorrenti in modo diretto e intenso, anche attraverso strumenti competitivi non convenzionali. L'autonomia, infine, è intesa come la capacità di un individuo o di un gruppo di agire in modo indipendente al fine di realizzare un'idea o una visione.

In linea teorica l'orientamento strategico finalizzato alla creazione di valore e al continuo perseguimento di opportunità attraverso attività innovative ben si adatta anche a organizzazioni che non siano orientate al profitto, quali le organizzazioni pubbliche. Tuttavia, le evidenze empiriche in questo settore sono ancora limitate e le potenzialità di applicazione del costrutto in tale ambito sono state solo parzialmente esplorate (ad esempio, Morris e Jones, 1999; Caruana et al., 2002; O'Shea et al., 2005; Bhuian et al., 2005; Morris et al., 2007). Inoltre, negli esempi riscontrati la misurazione dell'orientamento imprenditoriale non tiene conto delle peculiarità del campo di applicazione e, di conseguenza, non viene proposto alcun adattamento degli strumenti di rilevazione.

Nel caso specifico delle università le differenze esistenti rispetto alle imprese - in termini di obiettivi, organizzazione e sistemi di governance - rendono ancor più 
necessaria un'attenta riflessione sulle dimensioni rilevanti attraverso cui valutare l'orientamento imprenditoriale. A partire da questa considerazione, un recente contributo di Todorovic et al. (2011) ha mirato a sviluppare una innovativa scala, concepita ad hoc per essere applicata al contesto delle università pubbliche e validata con successo in Canada. Gli item inseriti nella scala, denominata dagli autori "Entre-U", sono il frutto d'interviste in profondità realizzate con quaranta docenti di quattro università canadesi. Agli intervistati - in buona parte direttori di dipartimento - è stato chiesto d'identificare gli elementi più rilevanti che nella loro percezione fossero riconducibili all'orientamento imprenditoriale di un dipartimento universitario, nonché evidenziare i vantaggi e gli svantaggi dell'essere imprenditoriale. Il numero di item è stato poi ridotto re-intervistando gli stessi docenti e chiedendo di indicare quelli più significativi e, in una seconda fase, attraverso un focus group con altri direttori di dipartimento. Alla fine del processo sono stati selezionati 23 item ricondotti a quattro dimensioni, totalmente diverse da quelle utilizzate in letteratura per misurare l'orientamento imprenditoriale delle imprese orientate al profitto. Tutti gli item sono stati, infatti, sviluppati con specifico riferimento alle università pubbliche, in considerazione degli obiettivi e della struttura organizzativa di tali entità. Non esiste pertanto alcuna sovrapposizione con gli item utilizzati tradizionalmente negli studi focalizzati sulle imprese.

Le dimensioni della scala Entre-U sono, infatti, le seguenti: research mobilization; unconventionality; industry collaboration; university policies. La prima dimensione attiene alle attività di ricerca realizzate nel dipartimento in termini sia di focus che di orientamento verso gli stakeholder esterni. La denominazione utilizzata dagli autori è finalizzata proprio a cogliere la capacità di "engage external stakeholders at all stages of research process, especially in making sure that research outcomes are communicated to multiple audiences in ways that are easily understood, so the results are more readily transferred and applied" (Todorovic et al., 2011). La seconda dimensione è inerente alla capacità del dipartimento di identificare nuove opportunità al di fuori del tradizionale ambiente accademico, privilegiando approcci non convenzionali nella ricerca di finanziamenti, nella risoluzione dei problemi, nella collaborazione con soggetti esterni al mondo universitario e così via. La terza dimensione valuta il grado di cooperazione del dipartimento con le imprese, a diversi livelli: coinvolgimento dei docenti, degli studenti e della struttura dipartimentale nel suo complesso. La quarta e ultima dimensione si riferisce alla percezione che il direttore di dipartimento ha delle politiche universitarie centrali e della misura in cui esse ostacolano o agevolano i dipartimenti nella propria azione innovativa e anticonvenzionale.

Attraverso un'indagine esplorativa, che ha coinvolto circa 180 direttori di dipartimento di vari atenei canadesi, è stata testata l'affidabilità della scala Entre-U nella misurazione delle performance imprenditoriali dei dipartimenti universitari, espresse in termini di brevetti e spin-off. Le dimensioni identificate sono effettivamente in grado di "spiegare" la capacità dei dipartimenti canadesi di valorizzare i risultati della propria ricerca, fornendo una puntuale definizione di orientamento imprenditoriale in quello specifico contesto. 
Tuttavia, poiché, come evidenziato in precedenza, sia le condizioni macroambientali che quelle interne alle singole istituzioni influenzano notevolmente la propensione e i risultati imprenditoriali delle università, ci è apparso interessante verificare in che misura le dimensioni della scala Entre-U risultano efficaci in un contesto completamente differente, come quello italiano. In linea con le finalità tipiche dei replication studies, il presente lavoro adotta lo stesso approccio metodologico dello studio esplorativo condotto in Canada nella misurazione dell'imprenditorialità e delle performance dei dipartimenti e mira a comparare i risultati ottenuti in un ambiente di ricerca completamente differente. L'obiettivo è cercare di comprendere in che misura le condizioni di contesto possano influenzare la relazione tra le variabili indagate.

\section{Metodologia della ricerca}

\subsection{Raccolta dei dati e caratteristiche del campione}

Un questionario strutturato è stato inviato ai direttori di dipartimento delle università italiane con i più significativi risultati imprenditoriali, espressi in termini di numero di brevetti e spin-off. A partire da dati secondari sulle attività di valorizzazione della ricerca da parte degli atenei - tra gli altri il rapporto annuale Netval (2011) - sono state selezionate le università che hanno fatto registrare le migliori performance. In seguito, sono stati contattati gli uffici per il trasferimento tecnologico di ciascun ateneo ed è stata richiesta la collaborazione per identificare $\mathrm{i}$ dipartimenti maggiormente coinvolti nelle attività commerciali. Si è quindi provveduto a contattare i direttori dei dipartimenti, dapprima telefonicamente e poi via $e$-mail. Acquisita la disponibilità a prendere parte all'indagine, è stato inviato tramite e-mail il link al questionario realizzato on line. Come incentivo alla partecipazione è stato garantito ai rispondenti l'anonimato e offerta la possibilità di visionare i risultati finali dell'indagine. Si sono naturalmente resi necessari ulteriori contatti per sollecitare la compilazione del questionario e validare alcune risposte.

Sono stati contattati complessivamente 251 dipartimenti, nel periodo compreso tra Gennaio e Maggio 2012. Di questi, 238 sono i direttori che hanno dato la disponibilità a ricevere il link al questionario e 113 quelli che hanno restituito il questionario compilato - con un tasso di risposta pari al 47,47\%. Tuttavia, ai fini dell'analisi, è stato necessario escludere 10 risposte a causa dell'incompletezza o della mancanza di dati. Pertanto il campione ottenuto risulta composto da 103 dipartimenti di 32 università (Tabella 1).

Il $31 \%$ circa dei dipartimenti afferisce all'area di Ingegneria; il $16 \%$ alle aree di Chimica e Medicina; il 10\% all'area di Fisica e Matematica; 1'8\% all'area di Biologia; il $4 \%$ all'area di Economia e il $12 \%$ ad aree scientifiche di diversa tipologia (ad esempio, scienze dell'antichità, agraria, architettura, scienze giuridiche etc.). 
Quanto alle dimensioni, la maggioranza del campione (53\%) presenta un numero di afferenti compreso nell'intervallo 31-60; il 20\% del campione appartiene alla classe 61-90 afferenti; il 16\% appartiene alla classe 15-30 afferenti; il 10\% appartiene alla classe 91-120 afferenti e soltanto 1'1\% del campione presenta un numero di afferenti superiore a 120 .

Infine, un'analisi descrittiva riguardo all'anno di costituzione mostra la presenza di dipartimenti nati in differenti epoche storiche - con il più "datato" che risale al 1969 e il più "recente" che è stato costituito negli ultimi mesi del 2011. Significativa è risultata la presenza di strutture dipartimentali costituite nell'anno solare antecedente l'indagine - dato questo ascrivibile agli effetti della riorganizzazione conseguente all'ultima Legge di Riforma dell'università, la n. 240/2010. Questa peculiarità ha avuto effetti anche sul tasso di risposta ottenuto, in quanto molte strutture risultavano ancora in fase di riorganizzazione nel momento in cui sono state contattate.

Tab. 1: Atenei selezionati e rispettivi dipartimenti contattati

\begin{tabular}{|c|c|}
\hline Atenei identificati & $\mathbf{N}^{\circ}$ Dipartimenti contattati \\
\hline Politecnico di Bari & 5 \\
\hline Politecnico di Milano & 3 \\
\hline Politecnico di Torino & 5 \\
\hline Scuola Superiore Sant'Anna di Pisa & 8 \\
\hline Università Bicocca di Milano & 8 \\
\hline Università de L'Aquila & 6 \\
\hline Università del Piemonte Orientale & 4 \\
\hline Università del Salento & 6 \\
\hline Università del Sannio & 2 \\
\hline Università della Calabria & 8 \\
\hline Università di Bologna & 7 \\
\hline Università di Brescia & 3 \\
\hline Università di Cagliari & 4 \\
\hline Università di Catania & 6 \\
\hline Università di Ferrara & 15 \\
\hline Università di Firenze & 4 \\
\hline Università di Genova & 3 \\
\hline Università di Modena e Reggio Emilia & 15 \\
\hline Università di Padova & 16 \\
\hline Università di Parma & 9 \\
\hline Università di Pavia & 6 \\
\hline Università di Perugia & 18 \\
\hline Università di Pisa & 20 \\
\hline Università di Sassari & 7 \\
\hline Università di Siena & 6 \\
\hline Università di Torino & 12 \\
\hline Università di Trieste & 4 \\
\hline Università di Udine & 7 \\
\hline Università Federico II di Napoli & 7 \\
\hline Università La Sapienza di Roma & 10 \\
\hline Università Politecnica delle Marche & 5 \\
\hline Università Tor Vergata di Roma & 12 \\
\hline TOTALE & 251 \\
\hline
\end{tabular}

Fonte: ns. elaborazioni 


\subsection{Variabili considerate}

Il questionario somministrato ai direttori di dipartimento è stato sviluppato a partire dallo studio pioneristico condotto in Canada, con gli adattamenti che si sono resi necessari, ed ha consentito di raccogliere informazioni: sulle quattro dimensioni dell'orientamento imprenditoriale, che costituiscono le variabili indipendenti della nostra indagine; sui risultati conseguiti dai dipartimenti per la valorizzazione della propria ricerca, che costituiscono le variabili dipendenti; e infine sulle caratteristiche del dipartimento, che rappresentano le variabili di controllo dell'indagine.

\section{Tab. 2: Item utilizzati per valutare l'orientamento imprenditoriale}

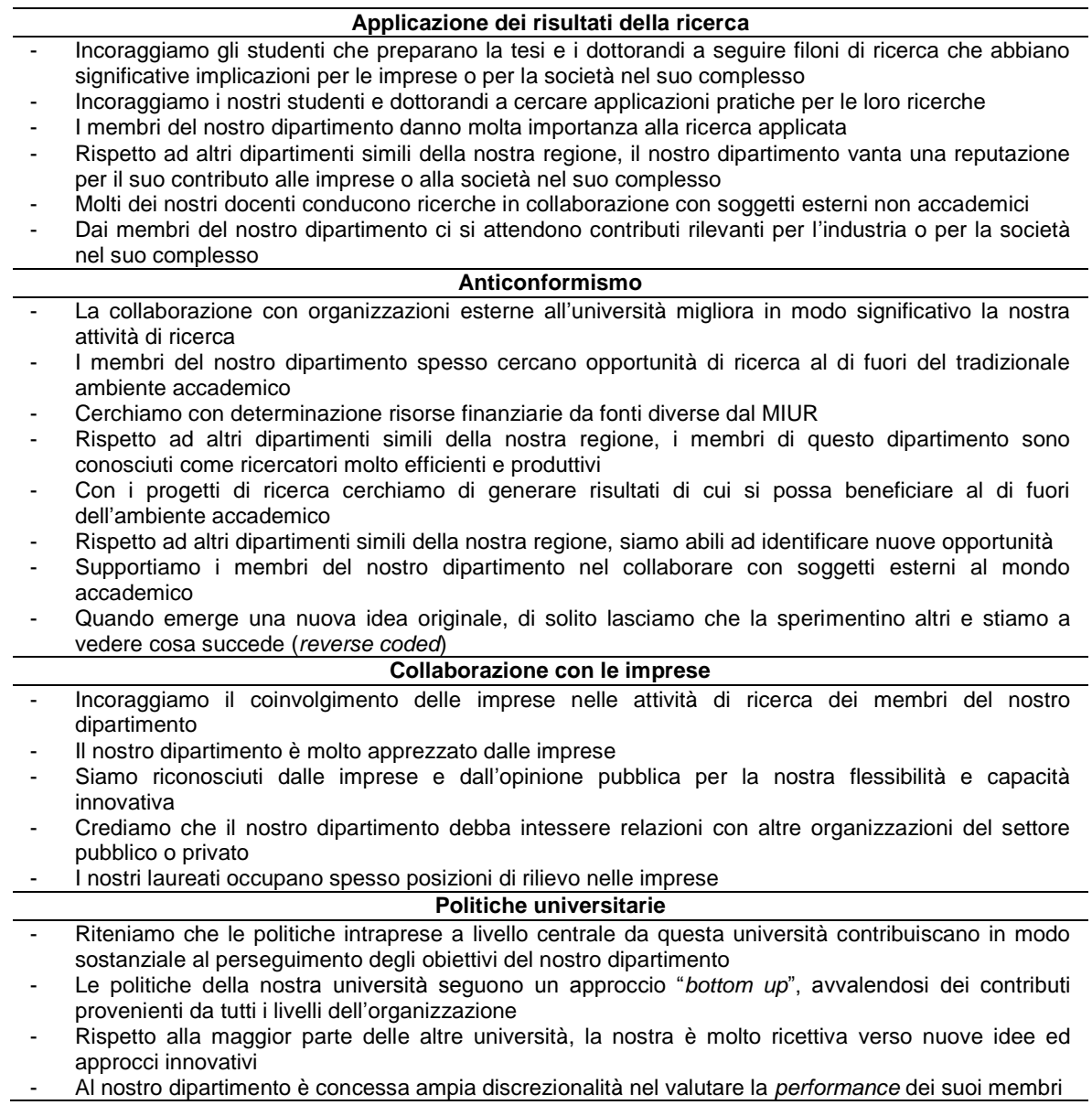

Fonte: ns. adattamento da Todorovic et al., (2011). 
Per misurare l'orientamento imprenditoriale si è partiti dai 23 item proposti da Todorovic et al. (2011), opportunamente tradotti e adattati. Rispetto a ciascuno di essi è stato richiesto ai direttori intervistati di esprimere il proprio grado di accordo su una scala Likert a 7 posizioni. La Tabella 2 riporta gli item rientranti in ciascuna delle dimensioni indagate.

Anche per la misurazione delle performance dei dipartimenti sono state utilizzate le medesime variabili dello studio esplorativo condotto in Canada. In particolare, ai direttori intervistati è stato richiesto di indicare: il numero complessivo di brevetti detenuti e il numero complessivo di imprese spin-off create dal personale afferente al dipartimento. Anche se sono potenzialmente numerose le manifestazioni dell'orientamento imprenditoriale a livello accademico, i brevetti e gli spin-off rappresentano le attività di valorizzazione della ricerca maggiormente considerate in letteratura (si veda, ad esempio, Jaffe e Lerner, 2001; Etzkowitz, 2004; Bercovitz e Feldman, 2006; Baglieri, 2008; Mustilli et al., 2011), nonché quelle usualmente censite nei report specialistici (si veda, ad esempio, il rapporto italiano Netval o quello europeo ProTon Europe). Tuttavia, nel tentativo di ampliare la valutazione delle performance imprenditoriali dei dipartimenti italiani, sono state considerate due ulteriori variabili. In linea con altri studi nazionali (tra gli altri, Mustilli et al., 2011; Netval, 2011), ai direttori intervistati è stato richiesto di indicare anche il numero complessivo di licenze e/o opzioni concesse sui brevetti detenuti. Tale variabile è stata però esclusa dall'analisi a causa dell'elevato numero di risposte mancanti. Ai direttori è stato inoltre richiesto di esprimere su una scala Likert a 7 posizioni il proprio grado di accordo rispetto alla seguente affermazione: "Il nostro dipartimento ha un forte orientamento imprenditoriale". L'obiettivo era di ottenere una valutazione percettiva dell'effettivo grado di imprenditorialità dei dipartimenti per poter poi indagare l'impatto delle singole variabili indipendenti.

Infine, si è provveduto a considerare come variabili di controllo le caratteristiche descrittive del dipartimento: area scientifica di riferimento; numero complessivo di afferenti; anno di istituzione. Si è inteso in tal modo verificare in che misura tali connotazioni potessero contribuire a spiegare le differenze in termini di performance imprenditoriali tra i dipartimenti.

\section{Analisi e risultati}

Come fase preliminare all'analisi, è stata verificata l'attendibilità e l'affidabilità della scala utilizzata, mediante il coefficiente $\alpha$ di Cronbach, che descrive la coerenza interna di raggruppamenti di item - tanto maggiore quanto più il valore dell'indicatore è vicino a $1(0<\alpha<1)$. La verifica della coerenza interna di ogni subtest permette anche di conoscere e definire la validità di costrutto della scala. I risultati dell'attendibilità della scala sono stati ottimali, com'è possibile osservare dalla Tabella 3 , che evidenzia $\mathrm{i}$ valori in relazione a ciascuna delle dimensioni ed alla scala nel suo complesso, e che riporta, inoltre, le statistiche descrittive. 
Tab. 3: Misura della validità della scala utilizzata, per dimensioni

\begin{tabular}{|c|c|c|c|c|c|}
\hline Variabili & Media & Dev. Std. & Min & Max & $\alpha$ \\
\hline Applicazione dei risultati & 5,129 & 1,068 & 2,000 & 7,000 & 0,863 \\
\hline Anticonformismo & 5,416 & 0,853 & 2,375 & 6,875 & 0,783 \\
\hline $\begin{array}{l}\text { Collaborazione con le } \\
\text { imprese }\end{array}$ & 5,117 & 1,090 & 2,200 & 6,800 & 0,864 \\
\hline Politiche universitarie & 4,473 & 1,185 & 1,750 & 7,000 & 0,818 \\
\hline Entre-U (complessiva) & 4,155 & 1,406 & 1,000 & 7,000 & 0,914 \\
\hline
\end{tabular}

Fonte: ns. elaborazioni

Sempre in via preliminare è stata verificata la presenza di problemi di multicollinearità, mediante gli indici Variance Inflation Factor (VIF) e Condition Index. Con riferimento ai VIF, i valori risultano appena superiori a 1 , ben lontani dalle soglie critiche. Similmente, i Condition Index hanno restituito valori ottimali essendo il più elevato pari a 3,835, ben inferiore al limite critico di 30 . La Tabella 4 riporta i coefficienti di correlazione ed evidenzia come le quattro dimensioni utilizzate per misurare l'orientamento imprenditoriale dei dipartimenti risultino tutte correlate in maniera positiva e significativa tra loro. Inoltre, tutte le quattro dimensioni risultano positivamente e significativamente correlate con l'orientamento imprenditoriale percepito dai direttori. Infine, la variabile spin-off risulta correlata in maniera positiva con tre delle dimensioni considerate, ma significativamente solo con la dimensione collaborazione con le imprese, mentre risulta correlata in maniera negativa con le politiche universitarie. La variabile brevetti è significativamente correlata con tutte le dimensioni considerate, ad eccezione delle politiche universitarie.

Tab. 4: Matrice di correlazione

\begin{tabular}{|c|c|c|c|c|c|c|c|}
\hline Variabili & 1 & 2 & 3 & 4 & 5 & 6 & 7 \\
\hline 1. Applicazione dei risultati & 1 & & & & & & \\
\hline 2. Anticonformismo & 0,743 & 1 & & & & & \\
\hline 3. Collaborazione con le imprese & 0,662 & 0,656 & 1 & & & & \\
\hline 4. Politiche universitarie & 0,209 & 0,308 & 0,324 & 1 & & & \\
\hline 5. Orientamento imprenditoriale percepito & 0,766 & 0,867 & 0,855 & 0,610 & 1 & & \\
\hline 6. Brevetti & 0,258 & 0,398 & 0,336 & 0,134 & 0,376 & 1 & \\
\hline 7. Spin-off & 0,156 & 0,178 & 0,195 & $-0,005$ & 0,186 & 0,175 & 1 \\
\hline
\end{tabular}

Fonte: ns. elaborazioni

Il legame tra l'orientamento imprenditoriale e le performance è stato verificato mediante un'analisi di regressione multipla effettuata utilizzando il software SPSS Statistics 19. L'analisi ha evidenziato un ruolo molto marginale delle variabili di controllo, risultando significativo il solo legame tra dimensioni del dipartimento misurate, come detto, in termini di numero di afferenti - e spin-off.

Se considerato nella sua totalità, come costrutto unico, l'orientamento imprenditoriale dei dipartimenti risulta significativamente legato alle performance. In particolare, però, emerge un ruolo più rilevante dell'orientamento imprenditoriale 
nel determinare il numero di brevetti detenuti dai docenti afferenti ai dipartimenti rispetto alle imprese spin-off create (Tabella 5).

Tab. 5: Relazione tra orientamento imprenditoriale e performance

\begin{tabular}{|lcrr|}
\hline & $\begin{array}{c}\text { Orientamento } \\
\text { imprenditoriale } \\
\text { percepito }\end{array}$ & Spin-off & Brevetti \\
\hline Entre-U (complessiva) & $0,912^{\star *}$ & $0,529^{*}$ & $5,032^{\star *}$ \\
$\mathrm{R}^{2}$ & 0,278 & 0,030 & 0,134 \\
$\mathrm{ADJUSTED} \mathrm{R}$ & & $0,021^{* *}$ & $0,125^{\star *}$ \\
\hline $\mathrm{p}<0,001^{*} \mathrm{P}<0,05$ & $0,271^{* *}$ & & \\
\hline
\end{tabular}

Fonte: ns. elaborazioni

Come noto dalla letteratura sull'orientamento imprenditoriale, tuttavia, le singole dimensioni che compongono il costrutto possono avere un diverso ruolo nel determinare le performance (Lumpkin e Dess, 1996). Anche nel caso della scala Entre-U pare opportuno verificare empiricamente il ruolo delle singole dimensioni. La Tabella 6 mostra i risultati della regressione effettuata considerando le singole dimensioni dell'orientamento imprenditoriale come variabili indipendenti e il livello di orientamento imprenditoriale percepito dai direttori, il numero di spin-off e di brevetti come variabili dipendenti.

Tab. 6: Relazione tra dimensioni dell'orientamento imprenditoriale e performance

\begin{tabular}{|ccrr|}
\hline & $\begin{array}{c}\text { Orientamento } \\
\text { imprenditoriale } \\
\text { percepito }\end{array}$ & Spin-off & Brevetti \\
\hline Applicazione dei risultati & $1,656^{* *}$ & 0,140 & 1,618 \\
Anticonformismo & $2,616^{* *}$ & 0,298 & $5,219^{\star *}$ \\
Collaborazione con le imprese & $0,224^{*}$ & $0,441^{*}$ & 1,826 \\
Politiche universitarie & 0,165 & $-0,181$ & 0,173 \\
$\mathrm{R}^{2}$ & 0,758 & 0,038 & 0,158 \\
ADJUSTED R ${ }^{2}$ & $0,751^{* *}$ & $0,028^{* *}$ & $0,150^{* *}$ \\
\hline${ }^{* *} \mathrm{p}<0,001{ }^{*} \mathrm{P}<0,05$ & & & \\
\hline
\end{tabular}

Fonte: ns. elaborazioni

Come si evince dalla tabella, il numero di spin-off risulta particolarmente influenzato dal grado di collaborazione dei dipartimenti con le imprese. La capacità di produrre brevetti risulta invece fortemente influenzata dalla dimensione dell'anticonformismo. Se si considera invece la percezione che i direttori hanno dell'orientamento imprenditoriale complessivo del proprio dipartimento, il modello di regressione multipla evidenzia un ruolo significativo di tutte le dimensioni, ad eccezione delle politiche universitarie. Il peso più rilevante è in questo caso esercitato dalla capacità di applicazione dei risultati della ricerca e, ancora, dall'anticonformismo. 


\section{Discussione dei risultati}

A differenza di altri studi sul tema dell'università imprenditoriale, nel presente lavoro l'attenzione non è stata concentrata sulla dotazione di risorse interne e sulle innovazioni di carattere organizzativo da parte delle università o sulle caratteristiche dell'ambiente esterno capaci d'influenzare $i$ risultati conseguiti in termini di valorizzazione della ricerca. L'attenzione è stata invece focalizzata sull'orientamento strategico delle università, mutuando un approccio tipicamente utilizzato negli studi sulle imprese e mirando a indagarne il legame con le performance imprenditoriali. Tale prospettiva pare essere particolarmente interessante ancorché, al momento, maggiormente trascurata dalla letteratura. A tal fine si è deciso di replicare la metodologia d'analisi recentemente proposta da un gruppo di studiosi canadesi (Todorovic et al., 2011), con l'obiettivo di testare la relazione tra $\mathrm{i}$ costrutti teorici in un contesto territoriale completamente diverso secondo un approccio tipico dei replication studies.

I risultati conseguiti hanno evidenziato l'impatto che l'orientamento imprenditoriale ha sulla capacità dei dipartimenti di creare imprese spin-off e di conseguire brevetti, confermando in una certa misura le conclusioni dello studio esplorativo di Todorovic et al. (2011), ma anche evidenziando importanti differenze tra i due contesti di ricerca. L'orientamento imprenditoriale, misurato come costrutto unitario, influenza sia il numero di brevetti che di spin-off, anche se risulta maggiormente legato ai primi. Questo risultato non è sorprendente, poiché la brevettazione e la creazione di nuove imprese sono processi di valorizzazione completamente differenti che si pongono a un differente stadio evolutivo. Come notato in precedenza, il brevetto è spesso il presupposto per la creazione di uno spinoff (Etzkowitz, 2004; Compagno e Pittino, 2006). Poiché molti dei dipartimenti coinvolti nell'indagine hanno da poco avviato una profonda riflessione sui meccanismi attraverso cui promuovere una valorizzazione dei risultati delle proprie attività di ricerca, è plausibile pensare che l'azione imprenditoriale ancora trovi manifestazione soprattutto nei brevetti piuttosto che nella creazione di nuove imprese. A conferma di quanto appena evidenziato, è opportuno considerare che nello studio esplorativo condotto in Canada - dove le università hanno profonde $\mathrm{e}$ radicate tradizioni di sfruttamento commerciale della propria ricerca - il legame della scala utilizzata per misurare l'orientamento imprenditoriale con le due variabili dipendenti è opposto: più forte con gli spin-off e meno con i brevetti, che risultano un'attività probabilmente meno "imprenditoriale" in quel contesto.

Inoltre, a una più approfondita analisi, è emerso che non tutte le dimensioni dell'orientamento imprenditoriale rivestono lo stesso ruolo nel determinare le performance dei dipartimenti universitari italiani. In particolare, per incrementare il numero di brevetti i risultati ottenuti sembrano indicare la necessità di puntare sull'anticonformismo, esplorando innovative modalità di interazione con gli stakeholder che possano migliorare le attività di ricerca e contribuire alla diversificazione delle fonti di finanziamento. Per incrementare la capacità di creare spin-off occorre invece dare maggior peso e investire maggiori risorse nella 
collaborazione con le imprese, puntando sulla flessibilità operativa e sul coinvolgimento diretto delle stesse nelle attività di ricerca dei membri del dipartimento. A differenza dello studio esplorativo realizzato in Canada, dunque, non tutte le dimensioni dell'orientamento imprenditoriale risultano essere significativamente legate alle variabili dipendenti. In particolare, la capacità di promuovere la ricerca applicata e le politiche universitarie non sembrano incidere direttamente sulle performance. Ancora una volta tali risultati non sorprendono e sono da ricondurre allo stadio evolutivo nel quale gli atenei coinvolti nell'indagine si trovano rispetto al modello "ideale" di università imprenditoriale.

Il ruolo marginale della dimensione che maggiormente attiene alla promozione della ricerca applicata è probabilmente ascrivibile alle caratteristiche delle università pubbliche italiane nelle quali, come notato da Varaldo (2010), «la terza missione [...] stenta a farsi luce ed è penalizzata [...] da tradizioni ideologiche e strutture di governance che sacrificano lo spirito innovativo e imprenditoriale degli ambienti più aperti e dinamici». La "seconda rivoluzione accademica" teorizzata da Etzkowitz (1998) è probabilmente nel nostro Paese ancora in divenire e il dibattito su come la terza missione possa coesistere con le due tradizionali missioni della didattica e della ricerca di base non sembra ancora mitigato. Allo stesso modo, il ruolo marginale delle politiche universitarie è riconducibile al fatto che ancora pochi sono gli atenei che hanno esplicitamente inglobato la terza missione nella propria strategia. Nei casi in cui si riscontrano risultati imprenditoriali significativi, le competenze e le attività di promozione della valorizzazione della ricerca sono concentrate in unità organizzative specializzate o nell'azione di singoli individui, piuttosto che diffuse in tutte le unità periferiche (Cesaroni et al., 2005; Piccaluga e Balderi, 2006; Riviezzo e Napolitano, 2010; Schillaci et al., 2008; Balderi et al., 2012). Come conseguenza, la nostra indagine sembra mostrare che in molte unità periferiche vi è la percezione che le politiche centrali non agevolino le attività di valorizzazione della ricerca.

Come noto dalla letteratura (Clark, 1998; Etzkowitz, 2004), la trasformazione dell'università verso il modello imprenditoriale è lenta e solitamente si sviluppa attraverso tre distinte fasi. All'inizio di questo processo di apprendimento organizzativo, l'università comincia a considerare in maniera strategica l'opportunità di essere maggiormente coinvolta con l'ambiente e comincia a specializzarsi in tale direzione. Successivamente assume un ruolo attivo nella commercializzazione di brevetti che derivano dalla propria ricerca e cerca di promuovere la creazione di spin-off. Nello stadio più avanzato assume un ruolo proattivo nel migliorare il sistema locale d'innovazione, spesso in collaborazione con le imprese e l'amministrazione pubblica. Pare possibile affermare che gli atenei italiani abbiano tutti superato la prima fase e siano, nella maggior parte, impegnati nella seconda fase, con poche eccezioni di università che sono già protagoniste delle dinamiche di sviluppo dei territori in cui sono inserite. In tal senso si giustificano i risultati ottenuti, che risentono di un processo evolutivo ancora "in progress".

Come più volte notato, solo recentemente i dipartimenti universitari italiani sono stati chiamati a riflettere operativamente su queste tematiche, soprattutto per effetto dell'istituzione di meccanismi di valutazione che non tengono conto più 
esclusivamente della qualità della didattica e della produttività scientifica, ma anche, seppur parzialmente, della capacità di applicare i risultati prodotti. In tal senso, è possibile che la scala di misurazione adottata - sviluppata e validata in un contesto molto più "maturo" da questo punto di vista - sia volta a leggere attività che correntemente i dipartimenti non sempre svolgono o che comunque stentano a ricondurre a un atteggiamento imprenditoriale. Sembrerebbe pertanto imprescindibile un'opportuna considerazione delle variabili ambientali nel tentativo di misurare empiricamente l'orientamento imprenditoriale dei dipartimenti universitari. Circostanza questa che emerge come una limitazione dello studio pioneristico che ha ispirato il presente lavoro e che rappresenta un'interessante direzione di sviluppo per il futuro.

Nonostante tali problematiche e pur considerando la limitata possibilità di generalizzare i risultati ottenuti - basati su un campione di 103 dipartimenti di 32 università italiane - sembrano emergere interessanti implicazioni dall'indagine. Rispetto al tradizionale approccio alla valutazione della capacità imprenditoriale delle università che si limita a considerare i risultati, ma spesso non ne indaga le determinanti, il presente lavoro sposta l'attenzione sull'orientamento strategico e individua i fattori sui quali occorre intervenire, a livello di singoli dipartimenti o di ateneo, per sostenere l'imprenditorialità accademica. In tal senso, sono identificate azioni concrete e buone pratiche che possono essere promosse per incrementare le attività di valorizzazione della ricerca scientifica, nelle sue diverse manifestazioni, in linea con le crescenti pressioni e aspettative di un maggiore coinvolgimento delle università nelle dinamiche di sviluppo dei territori in cui operano - specie nell'attuale momento storico.

\section{Limitazioni e future direzioni di ricerca}

L'indagine realizzata non è esente da limitazioni. Innanzitutto, la consistenza del campione ottenuto, come già evidenziato, non è elevata in senso assoluto, ma è in linea con altri studi sul tema e in senso relativo - con un tasso di risposta prossimo al $50 \%$ - può essere considerata soddisfacente.

Altra limitazione è ascrivibile al limitato potere esplicativo del modello di regressione sviluppato - come evidenziato dal valore del coefficiente $\mathrm{R}^{2}$ che, ancorché sempre significativo da un punto di vista statistico, ha restituito valori bassi. Inoltre, il modello sembra spiegare più efficacemente la differenza di risultati in termini di brevetti, ma solo limitatamente quella in termini di spin-off. Come evidenziato, ciò è imputabile allo strumento di rilevazione adottato, che probabilmente avrebbe richiesto un maggior adattamento degli item. Più in generale, occorre considerare che diverse sono le variabili che incidono sul livello di imprenditorialità accademica e l'orientamento strategico rappresenta soltanto uno dei fattori che sanciscono la differenza di performance tra i dipartimenti.

Similmente, occorre evidenziare il possibile effetto dell'approccio di ricerca sui risultati ottenuti. L'indagine è stata realizzata utilizzando un singolo key-informant, 
identificato nella persona del direttore di dipartimento, cui è stato indirizzato un questionario strutturato. Anche se tale approccio è largamente impiegato in letteratura e si è rivelato altamente affidabile in studi con obiettivi similari, sarebbe interessante integrare $\mathrm{i}$ dati percettivi con dati oggettivi, di fonte secondaria. In particolare, risulterebbe di estremo interesse integrare $\mathrm{i}$ dati di performance con ulteriori variabili (come, ad esempio, il numero di progetti di ricerca congiunti con le imprese, il numero di laureati impiegati, la presenza nell'offerta formativa di insegnamenti incentrati sull'imprenditorialità etc.), nonché integrare i dati sull'effettivo livello di orientamento imprenditoriale (considerando, ad esempio, il peso attribuito alla terza missione nei documenti strategici degli atenei e dei dipartimenti, quali statuto, regolamenti, declinazione della missione etc.). In tal senso s'intende procedere per sviluppare ulteriormente i risultati della ricerca.

Una menzione è necessaria anche per la tipologia di analisi statistica effettuata. Approcci differenti - quali modelli di equazioni strutturali - avrebbero probabilmente restituito risultati più puntuali. In questa ulteriore direzione ci si muoverà per futuri sviluppi della ricerca. Inoltre, al fine di verificare in che misura il contesto ambientale possa influire sui risultati e sulle relazioni esistenti tra le variabili indagate - come sembrerebbe emergere dal confronto tra i nostri risultati e quelli dello studio esplorativo condotto in Canada - appare interessante ipotizzare un'analisi comparativa tra più paesi, a partire da quelli nord-europei. E questa rappresenta un'ulteriore, interessante prospettiva per proseguire la ricerca.

\section{Bibliografia}

BAGLIERI D. (2008), "Brevetti universitari e trasferimento tecnologico: alcune considerazioni critiche", Sinergie, n. 75, pp. 175-193.

BALDERI C., DANIELE C., PICCALUGA A. (2012), "Gli uffici di trasferimento tecnologico delle università italiane: numeri ma non solo numeri", Economia e Politica Industriale, vol. 39, n. 4, pp. 135-159.

BARNETT R. (2003), Beyond all reason: Living with ideology in the university, The Society for Research into Higher Education \& Open University Press, Buckingham.

BENCARDINO F., NAPOLITANO M.R. (2011), "L'Università nei processi di sviluppo economico e sociale", in Bianchi A., (a cura di), Le Università del Mezzogiorno nella Storia dell'Italia Unita 1861-2011, SVIMEZ, Il Mulino, Bologna, pp. 123-134.

BERCOVITZ J., FELDMAN M. (2006), "Entrepreneurial universities and technology transfer: a conceptual framework for understanding knowledge-based economic development", Journal of Technology Transfer, vol. 31, n. 1, pp. 175-188.

BHUIAN S.N., MENGUC B., BELL S.J. (2005), "Just entrepreneurial enough: the moderating effect of entrepreneurship on the relationship between market orientation and performance ", Journal of Business Research, vol. 58, n. 1, pp. 9-17.

BOK D. (2003), Universities in the marketplace. The commercialization of higher education, Princeton University Press, Princeton.

CARUANA A., EWING M.T., RAMASESHAN B. (2002), "Effects of some environment challenges and centralization on the entrepreneurial orientation and performance of public sector entities", Service Industries Journal, vol. 22, n. 2, pp. 43-58. 
CESARONI F., CONTI G., PICCALUGA G., MASCARA P.C. (2005), "Technology Transfer Offices (TTO) in Italian Universities: What they do and how they do it", Working Paper, 5, IN-SAT Laboratory, Scuola Superiore S. Anna di Pisa.

CLARK B.R. (1998), Creating Entrepreneurial Universities. Organisational pathways of transformation, Pergamon Press, New York.

CNVSU (2011), Undicesimo Rapporto sullo Stato del Sistema Universitario, Comitato Nazionale per la Valutazione del Sistema Universitario, Ministero dell'Istruzione, dell'Università e della Ricerca, Roma.

CODA V. (1988), L'orientamento strategico dell'impresa, UTET, Torino.

COMPAGNO C., PITTINO D. (2006), Ricerca scientifica e nuove imprese. Spin-off accademici e valore della conoscenza, Isedi, Novara.

COVIN J.G., SLEVIN D.P. (1986), "The development and testing of an organizational-level entrepreneurship scale", in Ronstadt R., (a cura di), Frontiers of Entrepreneurship Research, Babson College, Wellesley.

ETZKOWITZ H. (1983), "Entrepreneurial Scientists and Entrepreneurial Universities in American Academic Science", Minerva, vol. 21, n. 2-3, pp. 198-233.

ETZKOWITZ H. (1998), "The norms of entrepreneurial science: cognitive effects of the new university-industry linkages", Research Policy, vol. 27, n. 8, pp. 823-834.

ETZKOWITZ H. (2004), "The evolution of the entrepreneurial university", International Journal of Technology and Globalisation, vol. 1, n. 1, pp. 64-77.

FELLER I. (1990), "Universities as engines of R\&D-based economic growth: they think they can", Research Policy, vol. 19, n. 4, pp. 335-348.

GEUNA A. (1998), "The internationalisation of the European university: A return to medieval roots", Minerva, vol. 36, n. 3, pp. 253-270.

GIBB A.A. (2005), "Towards the Entrepreneurial University. Entrepreneurship Education as a lever for change", NCGE Policy paper series, n. 15.

GRAS J.M.G., LAPERA D.R.G., SOLVES I.M., JOVER A.J.V., AZUAR J.S. (2008), “An empirical approach to the organisational determinants of spin-off creation in European universities", International Entrepreneurship and Management Journal, n. 4, pp. 187198.

GURDON M.A., SAMSOM K.J. (2010), “A longitudinal study of success and failure among scientist-started ventures", Technovation, vol. 30, n. 3, pp. 207-214.

HAYES D., WYNYARD R., (a cura di), (2002), The McDonaldization of higher education, Bergin \& Garvey, Westport.

HOYE K., PRIES F. (2009), “'Repeat commercializers,' the 'habitual entrepreneurs' of university-industry technology transfer", Technovation, vol. 29, n. 10, pp. 682-689.

HUSSLER C., PICARD F., TANG M.F. (2010), "Taking the ivory from the tower to coat the economic world: regional strategies to make science useful", Technovation, vol. 30, n. 9/10, pp. 508-518.

JAFFE A.B., LERNER J. (2001), "Reinventing public R\&D: patent policy and the commercialization of national laboratory technologies", The RAND Journal of Economics, vol. 32, n. 1, pp. 167-198.

KLOFSTEN M., JONES-EVANS D. (2000), "Comparing Academic Entrepreneurship in Europe-The Case of Sweden and Ireland", Small Business Economics, vol. 14, n. 4 , pp. 299-310.

LERNER J. (2005), "The University and the Start-Up: Lessons from the Past Two Decades", Journal of Technology Transfer, vol. 30, n. 1/2, pp. 49-56.

LIYANAGE S., MITCHELL H. (1994), "Strategic management of interactions at the academic-industry interface", Technovation, vol. 14, n. 10, pp. 641-655. 
LUMPKIN G.T., DESS G.G. (1996) "Clarifying the entrepreneurial orientation construct and linking it to performance ", The Academy of Management Review, vol. 21, n. 1, pp. 135-172.

MACHO-STADLER I., PEREZ-CASTRILlO D., VEUGELERS R. (2008), "Designing contracts for university spin-offs", Journal of Economics \& Management Strategy, vol. 17, n. 1, pp. 185-218.

MILLER D. (1983), "The correlates of entrepreneurship in three types of firms", Management Science, n. 29, pp. 770-791.

MORRIS M.H., COOMBES S., SCHINDEHUTTE M., ALLEN J. (2007), “Antecedents and outcomes of entrepreneurial and market orientations in a non-profit context: theoretical and empirical insights", Journal of Leadership and Organizational Studies, n. 13, pp. 12-39.

MORRIS M.H., JONES F.F. (1999), "Entrepreneurship in established organizations: the case of the public sector", Entrepreneurship Theory and Practice, vol. 24, n. 1, pp. 71-91.

MUSTILLI M., CAMPANELLA F., SORRENTINO F. (2011), "La valutazione delle performance innovative dei sistemi locali di innovazione: il caso dell'aerospazio e dei nuovi materiali in Campania”, Sinergie, n. 84, pp. 209-229.

NAPOLITANO M.R., RIVIEZZO A. (2008), "The institutional education and training for entrepreneurship development in the Italian universities", International Journal of Entrepreneurship and Innovation Management, vol. 8, n. 6, pp. 665-685.

NETVAL (2011), Potenziamo la catena del valore. Ottavo rapporto Netval sulla valorizzazione della ricerca nelle università italiane, Network per la valorizzazione della ricerca universitaria, Milano.

NETVAL (2012), Pronti per evolvere. Nono rapporto Netval sulla valorizzazione della ricerca pubblica italiana, Network per la valorizzazione della ricerca universitaria, Milano.

NETVAL (2013), Seminiamo ricerca per raccogliere innovazione. Decimo rapporto Netval sulla valorizzazione della ricerca pubblica italiana, Network per la valorizzazione della ricerca universitaria, Milano.

O'SHEA R.P., ALLEN T.J., CHEVALIER A., ROCHE F. (2005), "Entrepreneurial orientation, technology transfer and spinoff performance of U.S. Universities", Research Policy, vol. 34, n. 7, pp. 994-1009.

PICCALUGA A. (2001), La valorizzazione della ricerca scientifica, Franco Angeli, Milano.

PICCALUGA A., BALDERI C. (2006), "Consistenza ed evoluzione delle imprese spin-off della ricerca pubblica in Italia", presentato al workshop Finanza \& Innovazione, Milano, 25 Settembre.

POWERS J.B., McDOUGALL P.P. (2005), "University start-up formation and technology licensing with firms that go public: a resource-based view of academic entrepreneurship", Journal of Business Venturing, n. 20, pp. 291-311.

PRODAN I., DRNOVSEK M. (2010), "Conceptualizing academic-entrepreneurial intentions: an empirical test", Technovation, vol. 30, n. 5/6, pp. 332-347.

REBORA G. (2008), "L'orientamento strategico dell'università", in Airoldi G., Brunetti G., Corbetta G., Invernizzi G. (a cura di), Economia Aziendale \& Management: scritti in onore di Vittorio Coda, Egea, Milano.

RIVIEZZO A., NAPOLITANO M.R. (2010), "Italian universities and their third mission: a longitudinal analysis of the organization and education renewal towards the 'entrepreneurial university' model”, Industry \& Higher Education, n. 3, pp. 227-236. 
ROBERTS E.B., MALONE D.E. (1996), "Policies and structures for spinning off new companies from research and development organizations", $R \& D$ Management, $\mathrm{n}$. 26, pp. $17-48$

SCHILLACI C.E., ROMANO M., LONGO M.C. (2008), “Academic Entrepreneurship, University Incubator and Corporate Governance", Sinergie, n. 75, pp. 89-107.

SLAUGHTER S., LESLIE L.L. (1997), Academic capitalism: Politics, policies, and the entrepreneurial university, Johns Hopkins University Press, Baltimore.

TODOROVIC W.Z., MCNAUGHTON R.B., GUILD P.D. (2011), "ENTRE-U: an entrepreneurial orientation scale for universities", Technovation, vol. 31 , n. 1, pp. 128-137.

VAN BURG E., ROMME A.G.L., GILSING V.A., REYMEN I.M.M.J. (2008), "Creating university spin-offs: a science-based design perspective", The Journal of Product Innovation Management, vol. 25, n. 2, pp. 114-129.

VAN GEENHUIZEN M., SOETANTO D.P. (2009), "Academic spin-offs at different ages: a case study in search of key obstacles to growth", Technovation, vol. 29, n. 10, pp. 671-681.

VARALDO R. (2010), “Università di Stato: un modello che frena l'eccellenza”, La Stampa, 28 Luglio, p. 33.

VENKATRAMAN N. (1989),"Strategic Orientation of Business Enterprises: The Construct, Dimensionality and Measurement”, Management Science, vol. 35, n. 8, pp. 942-962. 